\title{
Impact of COVID-19 pandemic on frequency of clinical visits, performance of MRI studies, and therapeutic choices in a multiple sclerosis referral centre
}

\author{
Alvaro Cobo-Calvo ${ }^{1}$ - Ana Zabalza ${ }^{1}$. Jordi Río ${ }^{1} \cdot$ Georgina Arrambide ${ }^{1}$. Susana Otero-Romero ${ }^{1}$ Paula Tagliani ${ }^{1}$. \\ Simón Cárdenas-Robledo ${ }^{1} \cdot$ Mireia Castillo ${ }^{1}$. Carmen Espejo ${ }^{1} \cdot$ Marta Rodriguez $^{1} \cdot$ Pere Carbonell ${ }^{1}$. \\ Breogán Rodríguez ${ }^{1} \cdot$ Luciana Midaglia $^{1} \cdot$ Ángela Vidal-Jordana $^{1} \cdot$ Carmen Tur $^{1} \cdot$ Ingrid Galan $^{1} \cdot$ Joaquín Castillo $^{1}$. \\ Manuel Comabella ${ }^{1}$. Carlos Nos $^{1} \cdot$ Cristina Auger $^{2} \cdot$ Mar Tintoré $^{1} \cdot$ Àlex Rovira ${ }^{2} \cdot$ Xavier Montalban $^{1}$. \\ Jaume Sastre-Garriga ${ }^{1}$
}

Received: 23 November 2021 / Revised: 29 December 2021 / Accepted: 30 December 2021 / Published online: 10 January 2022

(c) The Author(s), under exclusive licence to Springer-Verlag GmbH Germany 2022

\begin{abstract}
Introduction To evaluate the impact of the COVID-19 pandemic on (1) number of clinical visits, (2) magnetic resonance (MR) scans, and (3) treatment prescriptions in a multiple sclerosis (MS) referral centre.

Methods Retrospective study covering January 2018 to May 2021.

Results The monthly mean (standard deviation [SD]) of visits performed in 2020 (814[137.6]) was similar to 2018 (741 [99.7]; $p=0.153$ ), and 2019 (797[116.3]; $p=0.747$ ). During the COVID-19 period (2020 year), 36.3\% of the activity was performed through telemedicine. The number of MR scans performed dropped by 76.6\% during the "first wave" (March 14 to June 21, 2020) compared to the mean monthly activity in 2020 (183.5[68.9]), with a recovery during the subsequent two months. The monthly mean of treatment prescriptions approved in 2020 (24.1[7.0]) was lower than in 2019 (30[7.0]; $p=0.049)$, but similar to 2018 (23.8[8.0]; $p=0.727)$. Natalizumab prescriptions increased in the "first wave" and onwards, whereas antiCD20 prescriptions decreased during the COVID-19 period.

Conclusion Maintenance of the number of clinical visits was likely due to telemedicine adoption. Although the number of MR dramatically dropped during the "first wave", an early recovery was observed. Treatment prescriptions suffered a slight quantitative decrease during 2020, whereas substantial qualitative changes were found in specific treatments.
\end{abstract}

Keywords Multiple sclerosis · COVID-19 $\cdot$ SARS-CoV-2 $\cdot$ Standards of care

\section{Introduction}

Xavier Montalban and Jaume Sastre-Garriga have contributed equally to this work.

Alvaro Cobo-Calvo

acobo@cem-cat.org

1 Centre d'Esclerosi Múltiple de Catalunya (Cemcat), Department of Neurology/Neuroimmunology, Edifici Cemcat, Vall d'Hebron Institut de Recerca, Hospital Universitari Vall d'Hebron, Universitat Autònoma de Barcelona, Pg. Vall d'Hebron 119-129, 08035 Barcelona, Spain

2 Section of Neuroradiology and Magnetic Resonance Unit, Department of Radiology (IDI), Vall d'Hebron Institut de Recerca, Hospital Universitari Vall d'Hebron, Universitat Autònoma de Barcelona, Barcelona, Spain
The novel coronavirus, SARS-CoV-2, causing coronavirus disease 2019 (COVID-19) has produced a rapidly expanding worldwide pandemic with a deep impact on all aspects of healthcare [1]. According to updated information, COVID-19 susceptibility and severity risk factors in patients with immune-mediated inflammatory diseases appear to be similar to the general population [2-6]. However, contradictory data exist on whether people with multiple sclerosis (MS) are at higher risk of being infected by SARS-CoV-2 and develop a poorer outcome than nonMS patients [7-10]. In addition, MS disease-modifying drugs (DMD) have been proved to be safe during the pandemic, except for anti-CD20 therapies or intravenous 
methylprednisolone that may increase the risk of infection and worsen COVID-19 evolution [7, 9, 11, 12].

COVID-19 has excessively strained healthcare systems all around the world [13], compelling neurology departments and MS centres to adopt deep structural changes to maintain standards of care [13-18]. To buffer the impact of COVID-19 on both clinical activity and treatment administration procedures, the MS Centre of Catalonia (Cemcat) implemented a large internal reorganization: organization of healthcare professionals into non-contact stand-alone teams, adoption of telemedicine, prioritization of diagnostic tests, changes in medicine dispensation, etc.[18]. As others did $[9,19,20]$, internal recommendations for the management of MS therapies were established, despite the unknown impact of DMD on the risk of COVID-19 susceptibility or outcomes at the beginning of the pandemic [18]. One year later, the evaluation of whether such urgent adaptations have enabled to keep standards of care in MS is warranted to face future extreme epidemiological situations.

The current study aims to assess the impact of the COVID-19 pandemic on (1) number of clinical visits, (2) magnetic resonance (MR) scans, and (3) treatment prescriptions in a MS referral centre.

\section{Methods}

The study was approved in the session Number 499 by the local Ethics Committee at Vall d'Hebron University Hospital (VHUH) in Barcelona, Spain.

\section{Research site}

Cemcat (Barcelona, Spain) is a specialized centre focusing on MS and other autoimmune disorders of the central nervous system (CNS). The centre provides healthcare coverage to patients with MS with a multidisciplinary approach based on neurological clinical visits, neuro-rehabilitation, neuropsychology, and a dedicated infusion centre. As a part of the Cemcat clinical activity, patients are routinely visited at an outpatient care centre every 3-12 months or at the time of new MS-related events. Intravenous acute or chronic treatments are administered at the infusion centre when medically needed. As part of the Cemcat supporting diagnostic tests activity, MR scans are performed at the Institut de Diagnostic per la Imatge (Section of Neuroradiology, VHUH), when needed. All potential DMD prescriptions are discussed in a weekly meeting attended by all involved treating neurologists, nurses, pharmacists and pharmacologists to reach consensus on any prescription issued.

\section{Patients and data collection}

This is a single-centre observational and retrospective study between January 2018 and May 2021. The number of first and follow-up visits at the outpatient care centre (clinical activity) was retrieved. Visits were classified into two types: face-to-face visits when patients moved to the outpatient care centre to be visited on-site, and telemedicine visits when the visit was performed by telephone or video call. The number of brain and spinal cord MR scans (radiological activity) was also retrieved. Both the number of visits and MR scans were anonymously extracted from the VHUH electronic database. The number and type of treatment prescription initiation (regardless of whether these were initiated in naïve or already treated patients-switches) were anonymously extracted from our Cemcat in-house clinical management software (eCemcat). Treatment prescriptions were classified into three groups: (1) first line (interferon, acetate glatiramer, teriflunomide, dimethyl-fumarate); (2) anti-CD 20 (rituximab, ocrelizumab); and (3) other second line (natalizumab, fingolimod, cladribine and alemtuzumab).

\section{Study periods}

Three different time periods were defined. The "COVID19" period corresponds to 2020 (January 1 to December 31, 2020). Two control periods were established: (1) A first "control period" (named 2018 year) comprising activity registered between January 1 to December 31, 2018, and (2) a second "control period" (named 2019 year) comprising activity registered between January 1 to December 31, 2019. The term "first wave period" was used to define the period of time between March 14, and June 21, 2020, in which a complete population lockdown was enforced by the Government of Spain [18]. Since in holiday periods, all activities tend to decrease, the term "holiday period" defines the period of time between July 15th to August 31st and December 20th to January 10th in every year. Finally, an "extension period" comprising January 1 to May 31, 2021, was included as sensitivity analysis to confirm trends of treatment prescriptions during 2020 in comparison to control periods, if any.

\section{Statistical analysis.}

Distribution of continuous variables (number of visits, MR scans and treatment prescriptions) was described as mean and standard deviation (SD). Categorical variables were described as number and percentage (\%).

Two different analyses were performed. First, the monthly number of clinical visits, MR scans and treatment prescriptions per period were compared between the control periods 
and the COVID-19 period using the unpaired Student's t test. Second, a dynamic description of the monthly number of clinical visits, MR scans and treatment prescriptions during the COVID-19 and control periods was detailed. To provide an objective tool to detect significant activity changes across periods, values beyond $\pm 2 \mathrm{SD}$ from the mean monthly activity observed during the whole study period (i.e., monthly number of visits, MR scans and treatment prescriptions) were defined as outliers; otherwise variables were considered to remain stable during the time on study. In all analyses, type I error was set at $p<0.05$. Statistical analyses and graphs were performed with STATA-12 software (64-bit, StataCorp, TX) and Prism 9 (9.01).

\section{Results}

\section{Clinical activity}

During the study period, a total of 28,230 visits were carried out at the outpatient care centre (8898 in 2018, 9564 in 2019, and 9768 in 2020) (Fig. 1a). No differences were found in the mean (SD) monthly number of visits performed in 2020 (814 [137.6]) compared to 2018 (741 [99.7]; $p=0.153$ ), and 2019 (797 [116.3]; $p=0.747)$. In 2020, out of 9768 of total visits, 6218 (63.7\%) visits were face-to-face and 3550 (36.3\%) were performed either by phone or videoconference; routine clinical care was only performed face to face in the control periods. When evaluating clinical activity dynamics, a slight decrease in the monthly number of visits occurred during the first two months of the "first wave period", although outlier

a

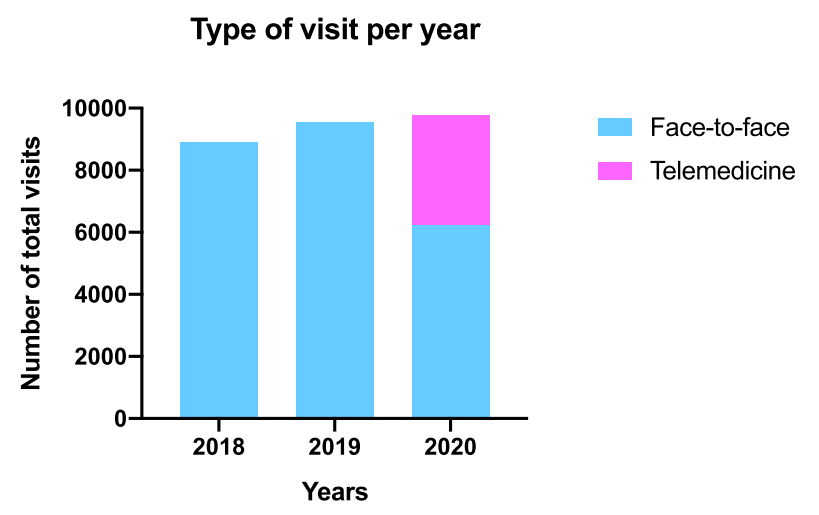

Fig. 1 Distribution of visits, according to 2020 year and control periods. a Total number of clinical visits in 2020, and control periods 2018 and 2019. b Longitudinal dynamics of the monthly number values were only observed in months including "holiday periods" (Fig. 1b).

\section{Radiological activity}

A total of 6765 MR scans were performed during the study period (4991 brain and 1774 spinal cord). MR scans were distributed as follows: 2207 during 2018, 2356 during 2019, and 2202 during 2020 (Fig. 2a). There were no differences in the mean (SD) monthly number of MR scans performed in 2020 (183.5 [SD 68.9]) compared to 2018 (183.9 [29.1]; $p=0.984)$ and 2019 (196 [17.5]; $p=0.538)$. When evaluating radiological activity dynamics, the monthly number of MR scans performed during the "first wave" decreased to reach an outlier value in April $2020(n=43)$, with a 76.6\% drop compared to the mean monthly activity in 2020 . A sharp increase was observed during the subsequent two months to reach an outlier high value in July $2020(n=289)$, a $58 \%$ increase (Fig. 2b).

\section{Treatment prescription activity}

The total number of treatment prescriptions was 925 throughout the whole study period (276 in 2018, 360 in 2019, and 289 in 2020) (Fig. 3a). The mean (SD) monthly number of treatment prescriptions in 2020 (24.1 [7.0]) was lower than in 2019 (30 [7.0]; $p=0.049)$, but similar to 2018 (23 [8.0]; $p=0.727$ ). A trend towards a lower mean (SD) number of treatment prescriptions was observed during the extension period (first 5 months of 2021) (23.2 [5.5]) when compared to $2019(p=0.072)$,

b

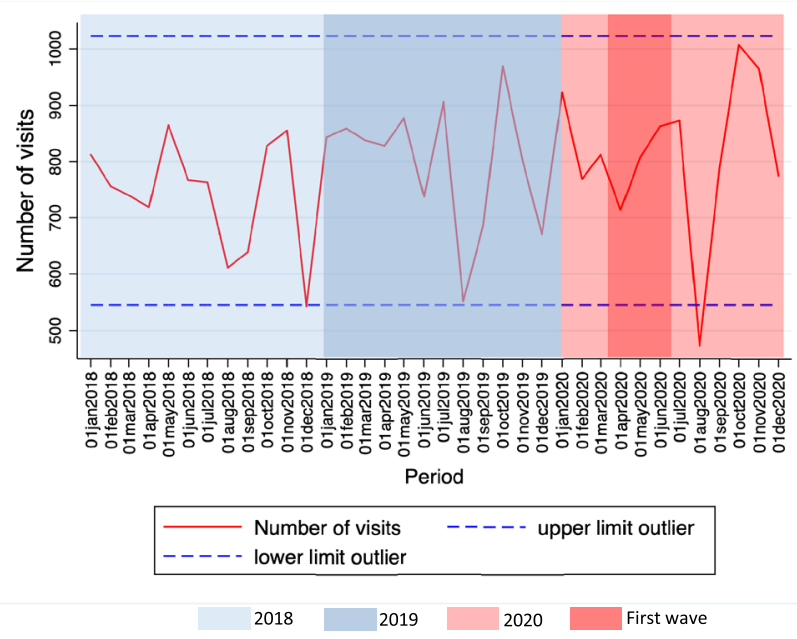

of visits during the study period. *Dash lines represent \pm 2 standard deviations the mean number of clinical visits during the whole study period 
a

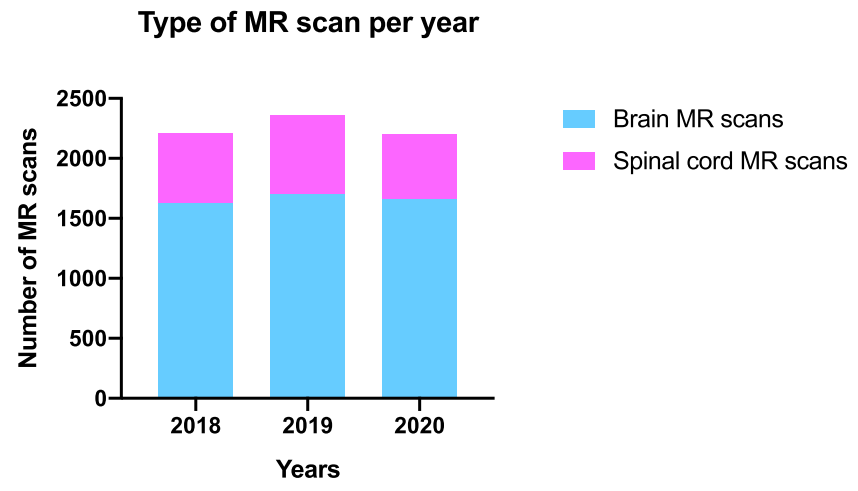

Fig. 2 Distribution of magnetic resonance scans, according to 2020 year and control periods. a Total number of magnetic resonance scans in 2020, and control periods 2018 and 2019. b Longitudinal

a

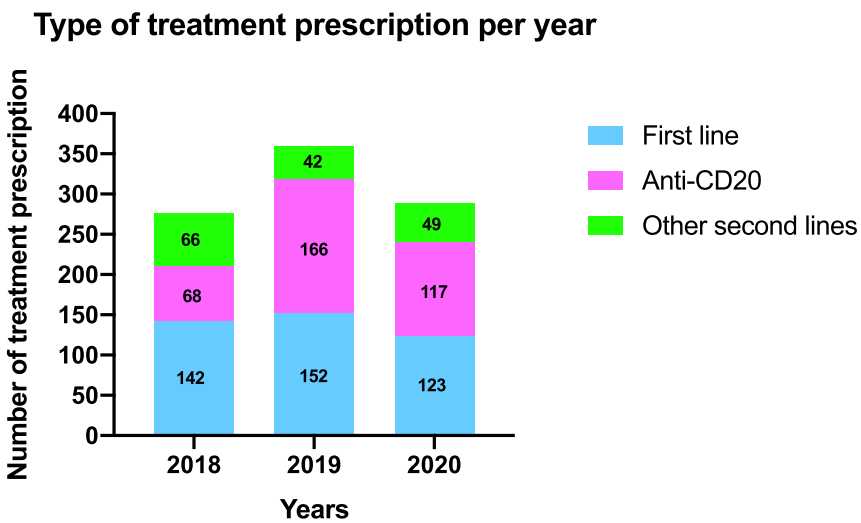

Fig. 3 Distribution of new treatment prescriptions, according to 2020 and control periods. a Total number of new treatment prescriptions in 2020, and control periods 2018 and 2019. b Longitudinal dynam-

but no differences with 2020 were observed $(p=0.805)$. Number treatment prescriptions are depicted in Table 1. When assessing treatment prescription dynamics, the monthly number of treatment prescriptions remained stable, with the exception of months including "holiday periods" (Fig. 3b). The monthly number of first-line treatment prescriptions remained stable throughout the study period (Fig. 4a). Anti-CD20 therapy prescriptions showed a continuous increase during 2019 to reach outlier value in October $2019(n=25 ; 146.4 \%$ increase compared to the mean of monthly anti-CD20 treatment prescriptions

\section{b}

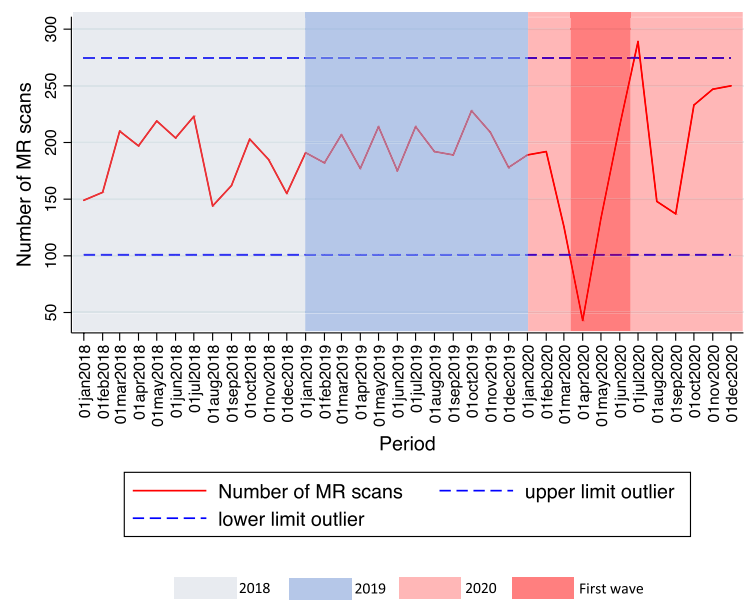

dynamics of the monthly number of magnetic resonances during the study period. *Dash lines represent \pm 2 standard deviations the mean number of magnetic resonance scans during the whole study period

b

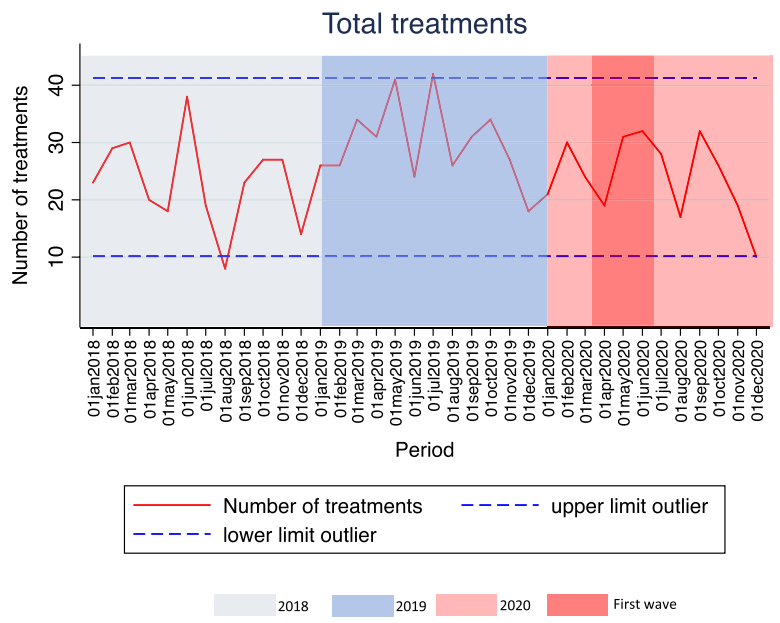

ics of the monthly number of new treatment prescriptions during the study period. *Dash lines represent \pm 2 standard deviations the mean number new treatment prescriptions during the whole study period

of the whole study period); thereafter a subsequent reduction was observed in 2020 and the first five months of 2021 (Fig. 4b). A decrease in the number of anti-CD20 treatment prescriptions was observed during the "first wave" ( $n=3$ prescriptions in April; $69.4 \%$ decrease compared to the mean of monthly treatment prescriptions of the whole study period), without reaching outlier values. The other second-line treatment prescriptions reached outlier values in June $(n=10)$ and October $2018(n=9)$, and remained stable during 2019 and 2020 (Fig. 4c). Finally, natalizumab experienced an incremental number of new 
Table 1 Number of monthly prescriptions of different therapy groups throughout the study period

\begin{tabular}{lcrrrr}
\hline Therapy & \multicolumn{1}{l}{ 2018 } & \multicolumn{1}{c}{2019} & \multicolumn{1}{c}{2020} & \multicolumn{1}{c}{$2021^{\mathrm{f}}$} & Whole study period $^{\mathrm{g}}$ \\
\hline First line $^{\mathrm{a}}$ & $11.8(5.3)$ & $12.7(4.67)$ & $10.3(3.91)$ & $11.2(4.02)$ & $11.6(4.64)$ \\
Anti-CD20 $^{\mathrm{b}}$ & $5.7(2.39)$ & $13.8(6.70)$ & $9.8(4.47)$ & $6.4(3.71)$ & $9.8(5.80)$ \\
Other second line $^{\mathrm{c}}$ & $5.5(2.64)$ & $3.5(1.51)$ & $4.1(1.92)$ & $5.6(2.07)$ & $4.4(2.19)$ \\
Natalizumab $^{\mathrm{d}}$ & $1.1(0.99)$ & $1.0(1.13)$ & $1.8(1.27)$ & $2.6(1.82)$ & $1.3(1.17)$ \\
All therapies $^{\mathrm{e}}$ & $23.0(7.97)$ & $30(6.98)$ & $24.1(6.99)$ & $23.2(45)$ & $25.7(7.77)$ \\
\hline
\end{tabular}

All reported values are: mean (standard deviation)

${ }^{a}$ First-line therapies include interferon, acetate glatiramer, teriflunomide, and dimethyl fumarate

${ }^{b}$ Anti-CD20 therapies include rituximab and ocrelizumab

${ }^{c}$ Other second-line therapies include natalizumab, fingolimod, cladribine, and alemtuzumab

${ }^{\mathrm{d}}$ Only natalizumab prescriptions

${ }^{\mathrm{e}}$ All therapies include first-line, anti-CD20 and other second-line therapies

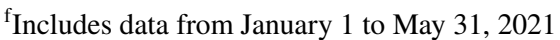

${ }^{\mathrm{g}}$ Includes data from 2018, 2019, and 2020

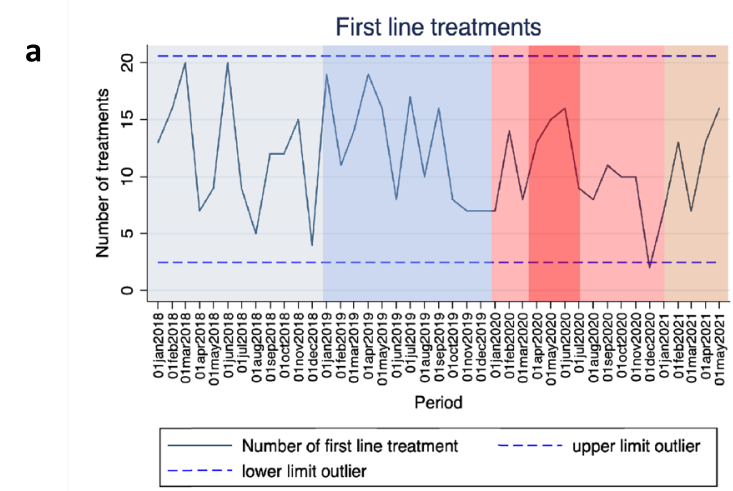

c

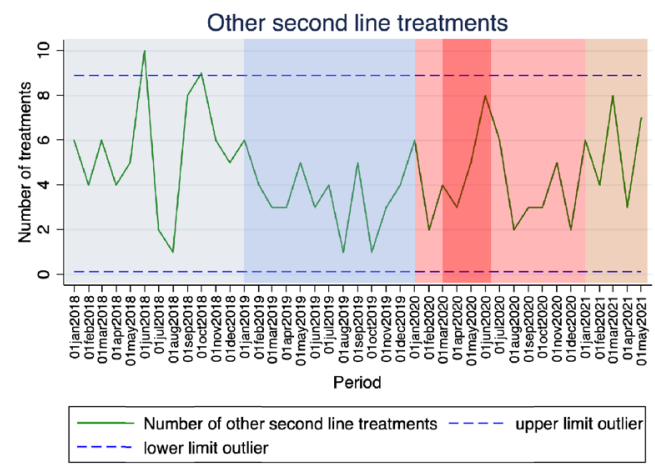

Fig. 4 Longitudinal dynamics of new treatment prescription, according to type of treatment prescription. a First-line treatments. b AntiCD20 drugs. c Other second-line treatment. d Natalizumab treatment. *In figure c, other second-line treatment comprises natalizumab,

prescriptions from the beginning of the COVID-19 pandemic to the end of the study period (Table 1); the monthly number of natalizumab prescriptions increased to reach
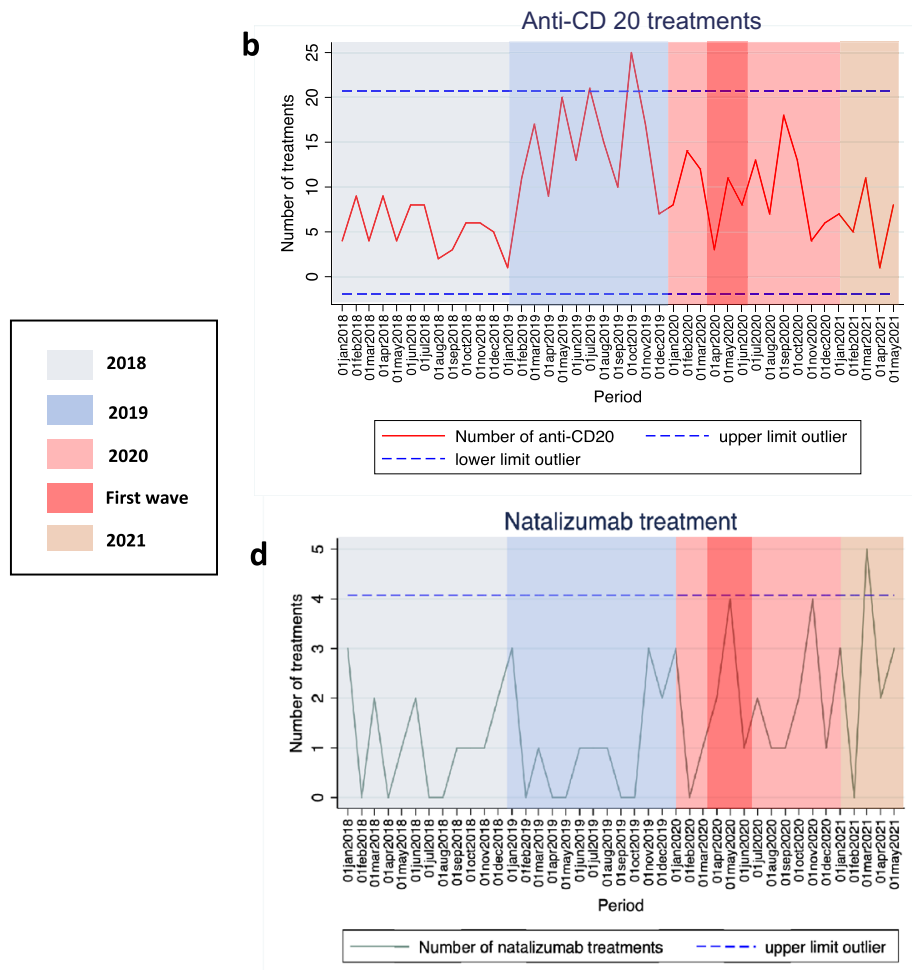

fingolimod, cladribine and alemtuzumab. **Dash lines represent \pm 2 standard deviations the mean number of new treatment prescriptions during the whole study period outlier value during the "first wave" $(n=4)$, in November $2020(n=4)$, and in March $2021(n=5)$ (Fig. 4d). All other 
second-line treatment prescriptions displayed stable patterns (data not shown).

\section{Discussion}

COVID-19 pandemic has hardly stricken formal operational plans in many health centres, leading to a negative impact in patient care. With the present study, we evaluate standards of care in a single MS centre for the first time, during the first year of COVID-19 pandemic. This may serve as a useful learning process for future pandemics and other potential local or global severe disruptions of usual operations. Overall, clinical and radiological activity was maintained as a result of successful adaptations to face the pandemic [18]. However, treatment strategies were subject to some variations which likely reflect a "change of concept" when treating MS patients during the COVID-19 pandemic.

The total number of clinical visits performed during 2020 remained stable in comparison with control periods, as the slight decrease observed during the "first wave" was rapidly corrected during the following months, thanks, at least in part, to rapid adoption of teleconsultation. Telemedicine had already been endorsed in terms of feasibility, cost-effectivity and patient satisfaction before the COVID-19 pandemic [21-24]. Interestingly, recent European and USA surveys have revealed that $73 \%$ of MS centres adopted telemedicine and one-third used telemedicine to provide over $75 \%$ of the clinical care during the first months of the pandemic, respectively $[25,26]$. These figures clearly highlight the impressive capability of many MS centres to develop rapid structural adaptations. At our centre, up to a third of the whole outpatient clinical visits was performed via telemedicine by the end of 2020. This lower figure of telemedicine adoption in our centre may be explained by that the fact that, even though 30 out of 79 (38\%) of Cemcat employees were either COVID-19-infected or quarantined in 2020, only $3(3.8 \%)$ of them were physicians; in this way, resorting to telemedicine was only driven by patient needs and not due to low availability of physicians on site. In addition, Cemcat facilities are located in a stand-alone building, with its own route of access, and away from other clinical facilities in the Hospital campus; such location may have decreased the perception of contagion risk, thus favoring face-to-face visits. Altogether, both the prompt adaptation from face-to-face towards telemedicine visits as well as the centre architectural particularities were essential points to keep clinical requirements during the COVID-19 pandemic.

Radiology departments have been greatly impacted by COVID-19. Preliminary data from Yale New Haven Hospital, USA, revealed volume imaging drops greater than $50 \%$ [27]. Another more recent international survey has shown that urgent MR scans were the only test allowed in $58 \%$ of centres, $17 \%$ of centres suspended or postponed radiological activity, and only $19 \%$ maintained usual activity [25]. At our centre, radiological activity suffered a 73\% abrupt reduction during the first two months of "the first wave" compared with overall activity in 2020 , but showed a sharp recovery immediately after. The radiology department went through a deep reorganization to guarantee a low transmission risk which directly determined radiological dynamics during the first months of pandemic: redeployment of technicians and radiologist to cover COVID-19 activity, more extended intervals between and longer duration of MR scans due to hygienic measures and, finally, an increase of examinations associated with COVID-19-related neurological disorders [28]. The overall stability of radiological activity observed across periods suggests that the initial activity decreased activity was not a consequence of variations in MR scan requests, but rather from rescheduling of non-urgent studies. Moreover, MR scan requests from primary care medicine were referred to other external centres, thus freeing slots to focus on radiological requests from our own centre, which greatly helped keeping the same levels of activity. Overall, the maintenance of the radiological activity after the first months of the pandemic indicates the adoption of first measures to be a key learning point to face the current or future pandemics.

MS therapy approach during the COVID-19 pandemic has been and still remains a challenge for MS neurologists trying to balance benefits and risks. Prescription of lympho-depleting agents in MS has been a matter of discussion due to the association with COVID-19 susceptibility and outcome risks $[7,29,30]$. Indeed, a survey has recently reported that $23 \%$ of centres avoided such therapies, whereas $8 \%$ postponed any type of DMD in treatment naïve-patients during the first months of the pandemic [25]. Description of treatment strategies performed during the first year of the pandemic might be of interest to conduct an interim assessment of previous recommendations at a time when the effects of DMD on COVID-19 susceptibility and outcomes were still unknown [18]. At our centre, treatment prescriptions during 2020 were lower than 2019, but similar to 2018 . Whether COVID-19 pandemic was the only reason for such decrease might be difficult to ascertain since treatment prescription patterns depend on several un-controlled temporally related variables: number of patients derived to the MS centre, changes in treatment guidelines, number of on-going clinical trials or a lower rate of treatment-switches related to a wider use of highly effective drugs, among others. Since both the total number of yearly visits and radiological tests were unaffected in 2020 but treatment prescriptions modified, it needs to be considered that the rapid and wide adoption of telemedicine might have modified treatment prescription by physicians, by a number of different reasons (i.e., missing relapses and progression events or delaying final 
decision till next face-to-face visit). A different relationship between neurologist and patient, a lack of direct physical examination or even the absence of important non-verbal information are some of the consequences of a sudden shift from in-person visits to telemedicine [31]. In fact, telemedicine may be better suited to other neurological diseases, such as epilepsy or headache, where the neurological examination is not a key point for decision-making [31]. This should not be interpreted as a claim against telemedicine in MS, but rather suggests that telemedicine could be more beneficial in patients with stable disease forms and lower chances of treatment switch.

A non-pandemic-related decrease in prescription of anti-CD20 drugs during 2020 compared to 2019 may have also had an impact on the overall number of treatment prescriptions. Anti-CD20 drugs were approved to treat progressive MS forms by the end of 2018, producing a high number of prescriptions during 2019 compared to 2018 . It is likely that important proportions of suitable patients for anti-CD20 drugs were treated during the first year after its approval, leaving therefore a small proportion of candidates to be treated during 2020. Obviously, a second potential cause for such decrease is the neurologist reluctance to prescribe anti-CD20 drugs due to the undesirable effects in the pandemic context $[18,32]$. Whether the COVID-19 vaccine will modify anti-CD20 prescription patterns is still unknown, although previous experience suggests a decremental humoral response to vaccines in anti-CD20 MS treated patients when compared to other DMD [33]. To this regard, most recent information points to an attenuated humoral response to COVID-19 vaccine in a non-negligible proportion of anti-CD $20 \mathrm{MS}$ patients [34, 35]. An interesting finding corroborating a "change of concept" on treatment strategies during the pandemic comes from the higher number natalizumab prescriptions once the pandemic hit MS patients, in comparison to previous years. These data present natalizumab to be a comfortable and safety option for patients with a highly active disease that may had otherwise been proposed to initiate anti-CD20 or other lymphocytedepleting drugs.

Because a pandemic cannot be foreseen, the nature of the present study entails those limitations associated with a retrospective design. In addition, some comments deserve to be added. First, the activity registered in a given health centre usually does not follow a linear pattern, but depends on un-controlled elements (i.e., disease incidence, hospital eligibility, course of the disease, etc.), which makes difficult to measure the direct impact of a pandemic on the clinical activity routine. As a proxy to identify pandemic-derived associations, outliers were defined as well as the inclusion of control periods before and after 2020, providing reasoned statements to consider these outliers as a direct consequence of the pandemic. Second, whether treatment prescriptions changes are a direct consequence of the COVID-19 pandemic or due to temporal fluctuations associated to intrinsic prescriptive patterns might be difficult to ascertain. Third, patients included in on-going clinical trials were not analyzed and, therefore, depicted figures do not show the whole spectrum of DMD used at our centre. Finally, the low sample sizes in some specific treatments prevented us to perform statistical comparisons. However, longitudinal descriptive analysis allowed us to show clear trends for anti-CD20 drugs or natalizumab.

\section{Conclusion}

Overall, the present study shows a proper adaptation of the clinical activity, even at a very early starting point of the pandemic by switching the type of visit from face-to-face towards telemedicine. Radiological activity was difficult to maintain during the first wave of pandemic, but showed an early recovery and further consolidation. Finally, overall treatment prescription suffered a slight quantitative decrease during 2020 together with substantial qualitative changes in prescription of specific treatments, although temporal fluctuations in prescription patterns may have affected these findings.

Funding The author(s) received no financial support for the research, authorship, and/or publication of this article.

\section{Declarations}

Conflicts of interest AC-C has received grant from Instituto de Salud Carlos III, Spain; JR19/00007; AZ has received travel expenses for scientific meetings from Biogen-Idec and Novartis, speaking honoraria from Eisai and a study grant from Novartis; JR has received speaking honoraria and personal compensation for participating on Advisory Boards from Almirall, Bayer Schering Healthcare, Biogen-Idec, Genzyme; GA has received compensation for consulting services or participation in advisory boards from Sanofi, Merck and Roche; research support from Novartis; travel expenses for scientific meetings from Novartis, Roche, Stendhal and ECTRIMS; speaking honoraria from Sanofi and Merck; and is a member of the International Women in Multiple Sclerosis (iWiMS) network executive committee; SO has received compensation for consulting services from Biogen-Idec and Genzyme, and research support from Novartis; PT was an ECTRIMS clinical fellowship awardee in 2019-2020; has received travel expenses for scientific meetings from Roche; SC-R was an ECTRIMS clinical fellowship awardee 2019-2020; has received travel expenses for scientific meetings from Biogen-Idec and Genzyme; compensation for consulting services or participation in advisory boards from Roche and Novartis; and speaking honoraria from Novartis. MC reports no disclosures. CE reports no disclosures; MR reports no disclosures; PC has received travel expenses from Biogen. PC's yearly salary is supported by a grant from Biogen to Fundació privada Cemcat towards statistical analysis; BR has received honoraria for consulting services from Wellspect; LM has received honoraria for consulting services and speaking honoraria from Roche and Novartis; AVJ has received compensation for consulting services and speaking honoraria from Novartis, Roche, Teva, Mylan, Biogen and Genzyme-Sanofi. CT is 
currently being funded by a Junior Leader La Caixa Fellowship. The fellowship code is LCF/BQ/PI20/11760008. She has also received the 2021 Fundación Merck Salud Award for the Investigation in Multiple Sclerosis (Spain). In 2015, she received an ECTRIMS Post-doctoral Research Fellowship and has received funding from the UK MS Society; IG reports no disclosures; JC reports no disclosures; MC has received compensation for consulting services and speaking honoraria from Bayer Schering Pharma, Merck Serono, Biogen-Idec, Teva Pharmaceuticals, Sanofi-Aventis and Novartis; $\mathrm{CN}$ has received funding for travel from Biogen-Idec and F. Hoffmann-La Roche, Ltd., and speaker honoraria from Novartis; CA reports no disclosures; MT is co-editor of Multiple Sclerosis Journal-Experimental, Translational and Clini$\mathrm{cal}$, and has received compensation for consulting services and speaking honoraria from Almirall, Bayer Schering Pharma, Biogen-Idec, Genzyme, Merck-Serono, Novartis, Roche, Sanofi-Aventis Viela-Bio and Teva Pharmaceuticals AR serves/ed on scientific advisory boards for Novartis, Sanofi-Genzyme, Synthetic MR, Bayer, Roche, Biogen, Icometrix and OLEA Medical, and has received speaker honoraria from Bayer, Sanofi-Genzyme, Bracco, Merck-Serono, Teva Pharmaceutical Industries Ltd, Novartis, Roche and Biogen. XM has received speaking honoraria and travel expenses for scientific meetings, has been a steering committee member of clinical trials or participated in advisory boards of clinical trials in the past 3 years with Actelion, Alexion, Bayer, Biogen, Celgene, EMD Serono, Genzyme, Immunic, Medday, Merck, Mylan, Nervgen, Novartis, Roche, Sanofi-Genzyme, Teva Pharmaceutical, TG Therapeutics, Excemed, MSIF and NMSS; JS-G has received compensation for consulting services and speaking honoraria from Almirall, Bayer, Biogen, Celgene, Sanofi, Merck, Novartis, Roche, Bial, Biopass and Teva, is member of the editorial committee of Multiple Sclerosis Journal, and director of Humoral and cellular responses to SARS-CoV-2 in convalescent COVID-19 patients with Multiple Sclerosis Revista de Neurología M.

Ethical approval The study was approved in the session Number 499 by the local Ethics Committee at Vall d'Hebron University Hospital (VHUH) in Barcelona, Spain.

Informed consent Written informed consent for participation was not required for this study.

\section{References}

1. Guan W, Ni Z, Hu Y et al (2020) Clinical characteristics of coronavirus disease 2019 in China. N Engl J Med 382:1708-1712. https://doi.org/10.1056/nejmoa2002032

2. Gianfrancesco M, Hyrich KL, Hyrich KL et al (2020) Characteristics associated with hospitalisation for COVID-19 in people with rheumatic disease: data from the COVID-19 global rheumatology alliance physician-reported registry. Ann Rheum Dis 79:859-866. https://doi.org/10.1136/annrheumdis-2020-217871

3. Aries P, Iking-Konert C (2020) No increased rate of SARS$\mathrm{CoV}-2$ infection for patients with inflammatory rheumatic diseases compared with the general population in the city of Hamburg (Germany). Ann Rheum Dis. https://doi.org/10.1136/annrh eumdis-2020-218400

4. Sarmiento-Monroy JC, Espinosa G, Londoño MC et al (2021) A multidisciplinary registry of patients with autoimmune and immune-mediated diseases with symptomatic COVID-19 from a single center. J Autoimmun 117:102580. https://doi.org/10.1016/j. jaut.2020.102580

5. Conticini E, Bargagli E, Bardelli M et al (2021) COVID-19 pneumonia in a large cohort of patients treated with biological and targeted synthetic antirheumatic drugs. Ann Rheum Dis 80(2):e14. https://doi.org/10.1136/annrheumdis-2020-217681

6. Michelena X, Borrell H, López-Corbeto M et al (2020) Incidence of COVID-19 in a cohort of adult and paediatric patients with rheumatic diseases treated with targeted biologic and synthetic disease-modifying anti-rheumatic drugs. Semin Arthritis Rheum 50:564-570. https://doi.org/10.1016/j.semarthrit.2020. 05.001

7. Zabalza A, Cárdenas-Robledo S, Tagliani P et al (2021) COVID19 in multiple sclerosis patients: susceptibility, severity risk factors and serological response. Eur J Neurol 28:3384-3395. https:// doi.org/10.1111/ene.14690

8. Louapre C, Collongues N, Stankoff B et al (2020) Clinical characteristics and outcomes in patients with coronavirus disease 2019 and multiple sclerosis. JAMA Neurol. https://doi.org/10.1001/ jamaneurol.2020.2581

9. Sormani MP, De Rossi N, Schiavetti I et al (2021) Disease-modifying therapies and coronavirus disease 2019 severity in multiple sclerosis. Ann Neurol. https://doi.org/10.1002/ana.26028

10. Sepúlveda M, Llufriu S, Martínez-Hernández E et al (2021) Incidence and Impact of COVID-19 in MS. Neurol Neuroimmunol Neuroinflamm 8:e954. https://doi.org/10.1212/NXI.0000000000 000954

11. Salter A, Fox RJ, Newsome SD et al (2021) Outcomes and risk factors associated with SARS-CoV-2 infection in a north American registry of patients with multiple sclerosis. JAMA Neurol 78:699-708. https://doi.org/10.1001/jamaneurol.2021.0688

12. Safavi F, Nourbakhsh B, Azimi AR (2020) B-cell depleting therapies may affect susceptibility to acute respiratory illness among patients with multiple sclerosis during the early COVID-19 epidemic in Iran. Mult Scler Relat Disord 43:102195. https://doi.org/ 10.1016/j.msard.2020.102195

13. Escalon MX, Herrera J (2020) Adapting to the COVID-19 pandemic in New York city. Am J Phys Med Rehab 99(6):453-458

14. Klein BC, Busis NA (2020) COVID-19 is catalyzing the adoption of teleneurology. Neurology 94:903-904

15. Baker D, Amor S, Kang AS et al (2020) The underpinning biology relating to multiple sclerosis disease modifying treatments during the COVID-19 pandemic. Mult Scler Relat Disord 43:102174. https://doi.org/10.1016/j.msard.2020.102174

16. Al-Jumah M, Abulaban A, Aggad $\mathrm{H}$ et al (2021) Managing multiple sclerosis in the Covid19 era: a review of the literature and consensus report from a panel of experts in Saudi Arabia. Mult Scler Relat Disord 51:102925. https://doi.org/10.1016/j.msard. 2021.102925

17. Krieger S (2020) COVID-19 will change MS care forever-commentary. Mult Scler J 26:1151-1152. https://doi.org/10.1177/ 1352458520935719

18. Sastre-Garriga J, Tintoré M, Montalban X (2020) Keeping standards of multiple sclerosis care through the COVID-19 pandemic. Mult Scler J 26(10):1153-1156. https://doi.org/10.1177/13524 58520931785

19. Costa-Frossard L, Moreno-Torres I, Meca-Lallana V, GarcíaDomínguez JM (2020) EMCAM (Multiple Sclerosis Autonomous Community of Madrid) document for the management of patients with multiple sclerosis during the SARS-CoV-2 pandemic. Rev Neurol 70:329-340

20. Brownlee W, Bourdette D, Broadley S et al (2020) Treating multiple sclerosis and neuromyelitis optica spectrum disorder during the COVID-19 pandemic. Neurology 94:949-952. https://doi.org/ 10.1212/WNL.0000000000009507

21. Robb JF, Hyland MH, Goodman AD (2019) Comparison of telemedicine versus in-person visits for persons with multiple sclerosis: a randomized crossover study of feasibility, cost, and satisfaction. Mult Scler Relat Disord 36:101258. https://doi.org/ 10.1016/j.msard.2019.05.001 
22. D'Haeseleer M, Eelen P, Sadeghi N et al (2020) Feasibility of real time internet-based teleconsultation in patients with multiple sclerosis: interventional pilot study. J Med Internet Res 22:e18178. https://doi.org/10.2196/18178

23. Bove R, Garcha P, Bevan CJ et al (2018) Clinic to in-home telemedicine reduces barriers to care for patients with MS or other neuroimmunologic conditions. Neurol Neuroimmunol Neuroinflamm 5:e505. https://doi.org/10.1212/NXI.0000000000000505

24. Sun S, Folarin AA, Ranjan Y et al (2020) Using smartphones and wearable devices to monitor behavioral changes during COVID19. J Med Internet Res 22:e19992. https://doi.org/10.2196/19992

25. Portaccio E, Fonderico M, Hemmer B et al (2021) Impact of COVID-19 on multiple sclerosis care and management: results from the European Committee for treatment and research in multiple sclerosis survey. Mult Scler J. https://doi.org/10.1177/13524 585211005339

26. Morrison EH, Michtich K, Hersh CM (2021) How the COVID19 pandemic has changed multiple sclerosis clinical practice: results of a nationwide provider survey. Mult Scler Relat Disord 51:102913. https://doi.org/10.1016/j.msard.2021.102913

27. Cavallo JJ, Forman HP (2020) The economic impact of the COVID-19 pandemic on radiology practices. Radiology 296(3):E141-E144. https://doi.org/10.1148/radiol.2020201495

28. Valdés P, Rovira A, Guerrero J et al (2020) Gestión de la pandemia. Visión del radiólogo. Radiologia 62:503-514. https://doi. org/10.1016/j.rx.2020.10.004

29. Arrambide G, Llaneza-González MÁ, Costa-Frossard França L et al (2021) SARS-CoV-2 infection in multiple sclerosis. Neurol
Neuroimmunol Neuroinflamm 8:e1024. https://doi.org/10.1212/ NXI.0000000000001024

30. Sormani MP, de Rossi N, Schiavetti I et al (2021) Disease-modifying therapies and coronavirus disease 2019 severity in multiple sclerosis. Ann Neurol 89:780-789. https://doi.org/10.1002/ana. 26028

31. Kristoffersen ES, Sandset EC, Winsvold BS et al (2021) Experiences of telemedicine in neurological out-patient clinics during the COVID-19 pandemic. Ann Clin Trans Neurol 8:440-447. https://doi.org/10.1002/acn3.51293

32. Reyes S, Cunningham AL, Kalincik T et al (2021) Update on the management of multiple sclerosis during the COVID-19 pandemic and post pandemic: an international consensus statement. J Neuroimmunol 357:577627. https://doi.org/10.1016/j.jneuroim.2021. 577627

33. Bar-Or A, Calkwood JC, Chognot C et al (2020) Effect of ocrelizumab on vaccine responses in patients with multiple sclerosis. Neurology 95:e1999-e2008. https://doi.org/10.1212/WNL.00000 00000010380

34. Ciotti JR, Valtcheva M, Cross AH (2020) Effects of MS diseasemodifying therapies on responses to vaccinations: a review. Mult Scler Relat Disord 45:102439. https://doi.org/10.1016/j.msard. 2020.102439

35. Achiron A, Mandel M, Dreyer-Alster S et al (2021) Humoral immune response to COVID-19 mRNA vaccine in patients with multiple sclerosis treated with high-efficacy disease-modifying therapies. Ther Adv Neurol Disord. https://doi.org/10.1177/17562 864211012835 